Solving the nonlocality riddle by conformal quantum geometrodynamics

This content has been downloaded from IOPscience. Please scroll down to see the full text. 2013 J. Phys.: Conf. Ser. 442012059

(http://iopscience.iop.org/1742-6596/442/1/012059)

View the table of contents for this issue, or go to the journal homepage for more

Download details:

IP Address: 5.172.32.172

This content was downloaded on 26/06/2016 at 10:09

Please note that terms and conditions apply. 


\title{
Solving the nonlocality riddle by conformal quantum geometrodynamics
}

\author{
Enrico Santamato \\ Dipartimento di Scienze Fisiche, Università di Napoli "Federico II", Complesso Universitario \\ di Monte S. Angelo, 80126 Napoli, Italy \\ E-mail: enrico.santamato@na.infn.it
}

Francesco De Martini

Accademia Nazionale dei Lincei, via della Lungara 10, 00165 Roma, Italy

E-mail: rancesco.demartini@uniroma1.it

\begin{abstract}
A rigorous $a b$ initio derivation of the quantum mechanics of a single particle with spin is presented starting by conformally Weyl-gauge-invariant principle. The particle is described as a relativistic top with six Euler's angles and quantum effects are introduced by assuming that the Weyl's curvature of the particle configuration space acts on it as an external scalar potential. Weyl's conformal covariance is made explicit in all steps of the theory. It is shown that metric of the configuration space accounts for non-quantum relativistic effects, while the affine connections account for quantum relativistic effects. In this way, classical and quantum features acquire well distinguished geometrical origin. A scalar wave function is also introduced to recover the connection with the standard quantum description based on Dirac's four-component spinors. Finally, the case of two entangled spins is considered in the nonrelativistic limit and it is found that the nonlocality rests on the entanglement of the spin internal orientational variables, playing the role of "hidden variables". The theory was carried out in the Minkowski space-time, but it can be easily extended to a space with nonzero Riemann curvature.
\end{abstract}

\section{Introduction}

Spin is a peculiar yet fundamental feature of the quantum world. Consequently, it is usually argued that no approach based on classical concepts is able to account for quantum spin. This seems particularly true for the spin $1 / 2$, because a classical system, even when quantized, can carry only integer values of angular momentum (in units of $\hbar$ ). In this work, we show that the mechanics of quantum spin may be derived from the mechanics of the relativistic spherical top in a conformally curved configuration space without having recourse to explicit quantization rules or operator algebra. Many attempts were made in the past to interpret quantum physics under the language of classical physics, dating from the hidden variables approach [1] to the MadelungBohm hydrodynamic approach $[2,3,4]$, to the Fényes-Nelson stochastic approach [5, 6] and others. The present work is based on the approach proposed some time ago to derive the Schrödinger [7, 8] and the Klein-Gordon [9] wave equations from space conformal geometry. Here beside space-time translational degrees of freedom, also orientational degrees of freedom 
have been added to the particle so to extend the theory to include spin $[10,11]$. The orientational coordinates are internal to the particle and play the role of hidden variables. In the case of two identical spatially distant spin $1 / 2$ particles, the results of the present theory are in full agreement with standard quantum mechanics and violation of Bell's inequalities $[12,13]$ is found, when the two particles are prepared in the EPR state [14]. Quantum correlations are shown to derive from nonseparable coupling among the internal angular variables of the two particles in the Weyl curvature and appear to be manifestly nonlocal. However, we studied the EPR entanglement in the nonrelativistic framework only, so we cannot say, at this stage of the theory, if this residual orientational coupling is really superluminal as some experiments seems to confirm [13].

Quantum effects are ascribed to the presence of a not trivial parallel transport law in the configuration space of the relativistic top. The metric plays no role and can be arbitrarily prescribed. However, we assume the Minkowsi metric with signature $(-,+,+,+)$. The particle motion and the affine connections for parallel transport are derived by a single action principle using Palatini's variation with fixed metric tensor. As a result, an integrable Weyl's connection is found and the theory acquires full invariance in a conformal change of the metric. The particle motion and the space affine connections must be consistent each other and they are part of a unique problem. The overall physical picture is analogous to the situation prevailing in the general relativity: geometry is not prescribed; rather it is determined by the physical reality. In turn, geometry acts as a "guidance field" for matter. However, although gravitational and quantum phenomena share a common geometrical origin, they rely on independent geometrical objects: the metric tensor for the former and the affine connections for the latter. As in the general relativity, the geometric approach forces to describe matter as a fluid or as a bundle of elementary trajectories in the configuration space rather than as a single point particle moving along one trajectory. In this respect, the Hamilton-Jacobi approach to mechanics plays a much more important role more than Newton's law in describing the particle motion.

\section{The conformal quantum geometrodynamics}

We consider a generic quantum system with $n$-dimensional configuration space $V_{n}$, spanned by generalized coordinates $q^{i}(i=1, \ldots, n)$. The space $V_{n}$ is endowed with a metric tensor $g_{i j}(q)$ that we assumed to be arbitrarily prescribed and with some affine connection fields $\Gamma_{j k}^{i}(q)$. The mechanics is governed by the multiple-integral variational principle

$$
\delta\left[\int d^{n} q \sqrt{g} \rho\left(g^{i j} \partial_{i} \sigma \partial_{j} \sigma+R_{W}\right)\right]
$$

where $g=\left|\operatorname{det}\left(g_{i j}\right)\right|, \rho(q)$ and $\sigma(q)$ are scalar fields and $R_{W}$ is the scalar curvature built up from the connections $\Gamma_{j k}^{i}(q)$. The variation with respect to $\rho(q), \sigma(q)$ yields, respectively [9]

$$
\begin{aligned}
g^{i j} \partial_{i} \sigma \partial_{j} \sigma+R_{W} & =0, \\
\frac{1}{\sqrt{g}} \partial_{i}\left(\sqrt{g} \rho g^{i j} \partial_{j} \sigma\right) & =0 .
\end{aligned}
$$

The variation of (1) with respect to the connections $\Gamma_{j k}^{i}(q)$ yields Weyl's conformal connection [9]

$$
\Gamma_{j k}^{i}=-\left\{\begin{array}{c}
i \\
j k
\end{array}\right\}+\delta_{j}^{i} \phi_{k}+\delta_{k}^{i} \phi_{j}+g_{j k} \phi^{i}
$$

where $\left\{\begin{array}{c}i \\ j k\end{array}\right\}$ are the Cristoffel symbols out of the metric $g_{i j}, \phi^{i}=g^{i l} \phi_{l}$, and $\phi_{i}$ is Weyl's vector given by

$$
\phi_{i}=-\frac{1}{n-2} \partial_{i}(\ln \rho)
$$


Equation (5) shows that the Weyl connection is integrable with Weyl's scalar potential $\phi$ given by $\phi=-\frac{1}{n-2} \ln \rho$. Equations $(2-5)$ are the main equations of the theory. From Eq. (5) we see that $\rho$ can be used in place of $\phi$ as Weyl's potential to calculate the affine connections. Thus, the scalar curvature derived from connections (4) can be written as

$$
R_{W}=R+\left(\frac{n-1}{n-2}\right)\left[\frac{g^{i j} \partial_{i} \rho \partial_{j} \rho}{\rho^{2}}-\frac{2 \partial_{i}\left(\sqrt{g} g^{i j} \partial_{j} \rho\right)}{\rho \sqrt{g}}\right] .
$$

The affine Weyl connections (4) are invariant under Weyl's conformal changes

$$
\begin{aligned}
g_{i j} & \rightarrow \lambda g_{i j}, \\
\phi_{i} & \rightarrow \phi_{i}-\frac{\partial_{i} \lambda}{2 \lambda} .
\end{aligned}
$$

Tensors $T$ which under Weyl's conformal gauge transformations (7) change as $T \rightarrow \lambda^{w(T)} T$ are said to transform simply. The exponent $w(T)$ is the Weyl weight of tensor $T$. Examples are the metric tensor with $w\left(g_{i j}\right)=1$ and Weyl's curvature $R_{W}$ with $w\left(R_{W}\right)=-1$. Weylgauge-invariant quantities as the affine connections (4) have $w=0$. The action principle (1) is Weyl-gauge invariant if $w(\sigma)=0$ and $w(\rho)=-(n-2) / 2$, which ensures Weyl-gauge invariance of all equations derived from this principle. Weyl's geometry is connected with the so-called co-covariant derivative $D_{i}$, defined as $D_{i} T=\nabla_{i} T-2 w(T) \phi_{i} T$, where $T$ is a tensor, $\nabla_{i}$ is the covariant derivative associated to the metric tensor $g_{i j}$, and $\phi_{i}$ is Weyl's vector. The metric tensor obeys $D_{i} g_{j k}=0$ and hence indices can be lowered and raised in the co-covariant derivative in Weyl's geometry as they are in the covariant derivative in Riemann's geometry. The Weyl-gauge covariance of Eqs. (2), (3), (5) is then made manifest by writing them as $D_{i} \sigma D^{i} \sigma+R_{W}=0$, $D_{i} D^{i} \sigma=0, D_{i} \rho=0$, respectively.

Equation (2) has the form of the classical Hamilton-Jacobi (HJ) equation with scalar potential $R_{W}$. We may then associate to the function $\sigma(q)$ the bundle of canonical trajectories defined by $d q^{i} / d \tau=\partial_{i} \sigma$, where $\tau$ is an arbitrary parameter. Each trajectory obeys the Euler-Lagrange equations derived by the Weyl-gauge invariant and parameter invariant single-integral variational principle

$$
\delta \int L d \tau=\delta\left[\int \sqrt{-R_{W} g_{i j} \dot{q}^{i} \dot{q}^{j}} d \tau\right]=0
$$

where the dot means derivative with respect to the parameter $\tau$. The HJ Eq. (2) and the variational principle (9) provide the mechanical features of the theory. These equations have the same form of the relativistic equations for a free particle, but the particle mass is replaced here by Weyl's curvature $R_{W}$ as required for Weyl-gauge invariance. The Lagrangian $L$ in Eq. (9) fully characterizes the system under study and usually provides the easiest way to find out the metric tensor $g_{i j}$.

Also Eq. (3) has a dynamical interpretation as continuity equation for the Weyl-gauge invariant current density $j^{i}$ given by

$$
j^{i}=\rho \partial_{i} \sigma \sqrt{g} .
$$

The continuity equation and the dependence of $R_{W}$ on $\rho$ shown in Eq. (6) are in agreement with the hydrodynamical picture of quantum mechanics first proposed by Madelung [2] and then developed by Bohm [3, 4]. In the Madelung-Bohm approach the particle trajectories are deterministically governed by classical mechanics and quantum effects are due to a "quantum potential" of quite mysterious origin, whose gradient acts as a newtonian force on the particle. According to the present approach the active potential originates from geometry, as does gravitation, and arises from the space curvature (6) due to the presence of the nontrivial affine connections (4) with Weyl's vector field given by Eq. (5). 
The particle dynamics (governed by $\sigma(q)$ ) and the affine connections (governed by $\rho(q)$ ) must be compatible, however. We must solve Eqs. (2) and (3) together. The nonlinear problem implied by Eqs. (2) and (3) looks very hard at first glance. However, by introducing the complex scalar function $\psi$ given by

$$
\psi(q)=\sqrt{\rho} e^{i \frac{S(q)}{\hbar}}
$$

with $S(q)=\xi \hbar \sigma(q)$, and

$$
\xi=\sqrt{\frac{n-2}{4(n-1)}},
$$

one converts Eqs. (2) and (3) into the linear differential equation

$$
\nabla_{k} \nabla^{k} \psi-\xi^{2} \hbar^{2} R \psi=0
$$

where $\nabla_{k} \nabla^{k}$ is the Laplace-Beltrami operator built from the metric tensor $g_{i j}$ and $R$ is the Riemann curvature appearing in Eq. (6). This is a striking result as it demonstrates that the Hamilton-Jacobi equation, applied to a general dynamical problem can be transformed into a linear wave equation, the foremost ingredient of the formal structure of quantum mechanics and of the Hilbert space theory. The value of $\xi$ given by Eq. (12) ensures that Eq. (13) is conformally invariant. The wave function is not gauge invariant, however, because its modulus has Weyl weight $w(|\psi|)=-(n-2) / 4$. The phase of $\psi$ is conformally invariant, instead. Equation (13) resembles the covariant quantum Klein-Gordon wave-equation with the mass term replaced by the Riemann curvature term $\hbar^{2} \xi^{2} R(q)$. What is more surprising is that any explicit reference to the Weyl vector field $\phi_{i}(q)$ and to the Weyl curvature $R_{W}$ has been cancelled out from Eq. (13). In fact, as said above, the curvature $R(q)$ and the covariant derivatives $\nabla_{i}$ in Eq. (13) are calculated using the Cristoffel symbols derived from the metric $g_{i j}$.

Finally, we observe that external electromagnetic vector fields $A_{i}(q)$ can be added to the theory by simply replacing $\partial_{i} \sigma$ with $\partial_{i} \sigma-A_{i}$ in all equations and adding a term $A_{i} \dot{q}^{i}$ to the Lagrangian in Eq. (9) so to include also invariance under electromagnetic gauge transformations $A_{i} \rightarrow A_{i}+\partial_{i} \chi$, $\sigma \rightarrow \sigma+\chi$. In this case, as expected, the wave equation (13) is modified by replacing the covariant derivative $\nabla_{i}$ with the operator $\nabla_{i}-A_{i}$ and the phase of the wave function (11) is affected by the electromagnetic gauge in the usual way. Therefore, Weyl's and electromagnetic gauges act separately on the modulus and phase of the wave function, respectively.

\section{The single spin $1 / 2$ particle}

In this section, we specialize the theory to the case of a single spin $1 / 2$ particle - one electron, say. We start considering the simplest model for the relativistic spinning particle, namely the spherical top described by six Euler angles, as made, for example, by Frenkel [15], Thomas [16] and in the classic work by Hanson and Regge [17]. Subsequent important works on relativistic spinning particles can be found in many textbooks $[18,19,20]$. Here, we simply imagine that the particle follows a path $x^{\mu}=x^{\mu}(\tau)$ in space-time, where $\tau$ is an arbitrary parameter along the path, and that it carries along with itself a moving fourleg $e_{a}^{\mu}=e_{a}^{\mu}(\tau)(\mu, a=0, \ldots, 3)$ with $e_{0}^{0}>0$. The fourleg vectors $e_{a}^{\mu}$ are normalized according to $g_{\mu \nu} e_{a}^{\mu} e_{b}^{\nu}=g_{a b}$ where $g_{\mu \nu}=g_{a b}=\operatorname{diag}(-1,1,1,1)$ is the Minkowski metric tensor. Setting $\Lambda=\left\{e_{a}^{\mu}\right\}$ and $G=\left\{g_{\mu \nu}\right\}$, the normalization relations can be cast in the matrix form as $\Lambda^{T} G \Lambda=G$, showing that the $4 \times 4$ matrix $\Lambda \in S O(3,1)$ is a proper orthochronous Lorentz matrix. The fourleg $e_{a}^{\mu}$ is parametrized by six Euler's angles $\theta^{A}=\{\alpha, \beta, \gamma ; \chi, \theta, \phi\}(A=1, \ldots, 6)$ : first three angles for space rotations and last three angles for boosts. Then, the configuration space of our relativistic top has $n=10$ coordinates: the four coordinates $x^{\mu}$ of the "center of mass" and the six Euler's angles $\theta^{A}$ for "orientation". The derivative of $e_{a}^{\mu}(\tau)$ with respect to $\tau$ can be written as $d e_{a}^{\mu} / d \tau=\omega_{\nu}^{\mu} e_{a}^{\nu}$. The contravariant tensor $\omega^{\mu \nu}=\omega_{\sigma}^{\mu} g^{\sigma \nu}$ is skewsymmetric and can be considered as the "angular 
velocity" of the top in space-time. The three space components of $\omega^{\mu \nu}$ are associated to rotations and the three time components to boosts.

The metric of the relativistic top configuration space is fixed by the Weyl-gauge and parameter invariant Lagrangian

$$
\begin{aligned}
L & =\sqrt{-R_{W}\left(g_{\mu \nu} \dot{x}^{\mu} \dot{x}^{\nu}+a^{2} g_{\mu \nu} g^{a b} \dot{e}_{a}^{\mu} \dot{e}_{b}^{\nu}\right)}= \\
& =\sqrt{-R_{W}\left(g_{\mu \nu} \dot{x}^{\mu} \dot{x}^{\nu}-a^{2} \omega_{\mu \nu} \omega^{\mu \nu}\right)} .
\end{aligned}
$$

When written in full as a function of the ten generalized coordinates $q^{i}=\left\{x^{\mu}, \theta^{A}\right\}$ and their derivatives, the Lagrangian (14) reduces to the canonical form of Eq. (9). In the presence of external electromagnetic fields a term $A_{\mu} \dot{x}^{\mu}+\kappa a^{2} F_{\mu \nu} \omega^{\mu \nu}$ with constant $\kappa$ must be added to the Lagrangian [10,11]. Once the metric is fixed by the dynamical system, we must find its dynamical state and compatible Weyl's connections. As said above, this is conveniently made by solving the wave equation (13). A long but straightforward calculation shows that the wave function $\psi(q)$ which solves Eq. (13) can be written as the Dirac product $\psi(q)=\bar{\Phi}(\theta) \Psi(x)=$ $\Phi^{\dagger}(\theta) \gamma^{0} \Psi(x)$, where $\gamma^{0}=\left(\begin{array}{ll}0 & 1 \\ 1 & 0\end{array}\right)$ is Dirac's matrix in the spinor representation and $\Phi(\theta)$ and $\Psi(x)$ are the four-component Dirac's spinors

$$
\begin{aligned}
& \Phi(\theta)=\left(\begin{array}{l}
D^{\sigma}(\theta) \\
D^{\dot{\sigma}}(\theta)
\end{array}\right), \\
& \Psi(x)=\left(\begin{array}{l}
\psi^{\sigma}(x) \\
\psi^{\dot{\sigma}}(x)
\end{array}\right) .
\end{aligned}
$$

Here $\psi^{\sigma}(x)$ and $\psi^{\dot{\sigma}}(x)$ are undotted and dotted two-component Lorentz spinors depending on the spacetime coordinates $x^{\mu}$ only, and $D^{\sigma}(\theta)$ and $D^{\dot{\sigma}}(\theta)$ are the first columns of the $D$-matrices belonging to the $(0,1 / 2)$ and $(1 / 2,0)$ irreducible representation of Lorentz group, respectively. These matrices are related by $D^{(0,1 / 2)}=\left[\left(D^{(1 / 2,0)}\right)^{\dagger}\right]^{-1}$ and depend on the Euler's angles $\theta^{A}$ only. To satisfy the wave equation, the four-component spinor $\Psi(x)$ must be a solution of the standard Klein-Gordon equation in space-time ${ }^{1}$ with mass $m$ given by

$$
m^{2}=\frac{3 \hbar^{2}}{2 a^{2} c^{2}}+\frac{6 \hbar^{2} \xi^{2}}{a^{2} c^{2}} .
$$

The first term in Eq. (17) comes from the spin (it vanishes for zero spin) and the second term comes from the constant Riemann curvature $R=6 / a^{2}$ of the configuration space. If we take $m=m_{e}$, the electron mass, the gyration radius $a$ of the associated relativistic top turns out to be of the order of the electron Compton length: $a \simeq \hbar /\left(m_{e} c\right)$. Notice that the spinor field $\Psi(x)$ has a constant mass in spite of the fact that in the Weyl-gauge invariant variational principle (1) and in the Lagrangian (14) Weyl's curvature field $R_{W}(q)$ replaces the mass. As it is well-known, the Klein-Gordon equation (and the second-order Dirac's equation) have unphysical four-component solutions, corresponding to a negative mass in the first-order Dirac's equation [21]. Any fourcomponent solution of the Klein-Gordon (or second-order Dirac) equation can be written as a superposition of solutions of the first-order Dirac's equation with positive and negative mass [11]. The latter must be discarded, which can be made also systematically [21]. Four-component field $\Psi(x)$ has been used here to preserve invariance under parity. However, the second-order Klein-Gordon (or Dirac) equation has also (left and right) chiral solutions corresponding to the two-component Lorentz spinor fields $\psi^{\sigma}(x)$ or $\psi^{\dot{\sigma}}$ in Eq. (16). As shown by Brown [22], the twocomponent solutions, beside reproducing the same physical result's of Dirac's equation when parity is restored, are also computationally easier to work with.

1 When electromagnetic fields are present, the Klein-Gordon equation is replaced by the second-order Dirac equation $[10,11]$. 


\section{The nonrelativistic limit}

A deeper insight into the scalar wave function $\phi(q)==\bar{\Phi}(\theta) \Psi(x)$ is obtained when the matrix $D(\theta)$ is factorized into the boost $B\left(e_{0}\right)$ associated to the time-like vector $e_{0}=\left\{e_{0}^{\mu}(\chi, \theta, \phi)\right\}$ of the particle fourleg and a rotation $R(\alpha, \beta, \gamma) \in \mathrm{SU}(2)$, viz $D(\theta)=B\left(e_{0}\right) R(\alpha, \beta, \gamma)$. The rotation $R$ belongs to the little Poincaré group of the time-like vector $e_{0}$ and, under generic Lorentz transformation $\Lambda$, transforms with the Wigner rotation $B\left(\bar{e}_{0}\right)^{-1} D(\Lambda) B\left(e_{0}\right)$, where $\bar{e}_{0}^{\mu}=\Lambda_{\nu}^{\mu} e_{0}^{\nu}$. According to our relativistic top picture, the rotation $R$ accounts for particle spin and the boost $B\left(e_{0}\right)$ accounts for the motion of the particle "center of energy" around the world line $x^{\mu}(\tau)$ of its "center-of-mass" [20, Chap. 20].

Having introduced the rotational degrees of freedom explicitly makes easy to go into the low velocity nonrelativistic limit of the theory. In this limit, the dotted and undotted Lorentz spinors $\psi^{\sigma}(x)$ and $\psi^{\dot{\sigma}}(x)$ coalesce into one two-component Pauli spinor $w^{\sigma}(x)$ and the fourleg angle $\chi \rightarrow 0^{2}$. Then, in the low velocity limit we obtain

$$
\begin{aligned}
\psi(q) & =R_{\sigma}^{-1}(\alpha, \beta, \gamma) w^{\sigma}(x)= \\
& =e^{\frac{i \gamma}{2}}\left(e^{\frac{i \alpha}{2}} \cos \frac{\beta}{2} w_{\uparrow}(\boldsymbol{r}, t)+e^{-\frac{i \alpha}{2}} \sin \frac{\beta}{2} w_{\downarrow}(\boldsymbol{r}, t)\right)
\end{aligned}
$$

where $R_{\sigma}^{-1}(\alpha, \beta, \gamma)$ is the first row of the inverse of the $\mathrm{SU}(2)$ rotation matrix $R(\alpha, \beta, \gamma)$. As expected, the two-component Pauli spinor $w^{\sigma}(x)=\left\{w_{\uparrow}, w_{\downarrow}\right\}$ obeys the non relativistic Schrödinger equation and its components correspond to spin up or down along the fixed $z$-axis, respectively [23]. To better see the role played by the wave function in the present approach, we consider the simple case of spin up state. From Eq. (18) with $w_{\downarrow}=0$ we calculate the mechanical action $S$ and the Weyl curvature $R_{W}$ as

$$
\begin{aligned}
S & =\frac{\hbar}{2}(\gamma+\alpha)+\arg \left(w_{\uparrow}(\boldsymbol{r}, t)\right), \\
R_{W} & =-\frac{5}{2 a^{2}(1+\cos \beta)}+R_{W}^{\uparrow}(\boldsymbol{r}, t)+\text { const. }
\end{aligned}
$$

where $R_{W}^{\uparrow}(\boldsymbol{r}, t)$ is the contribution of $w_{\uparrow}(\boldsymbol{r}, t)$ to Weyl's curvature. From Eq. (19) we see that the $\beta$ coordinate is cyclic, and, hence, $\beta$ is a constant of motion. From Eq. (20) we see that the particle is not free, but is subjected to a self-force proportional to the gradient of Weyl's curvature. This self-force has a geometric origin and cannot be eliminated: it is needed to have Weyl-gauge invariance. However, its existence is hidden in the standard quantum mechanics based on the space-time spinor $w_{\uparrow}(\boldsymbol{r}, t)$, which obeys the Schrödinger equation for the free particle. In the next section, we will demonstrate that the geometric force due to Weyl's curvature is the source of quantum entanglement and of the EPR paradox.

\section{The EPR paradox}

The calculation to obtain Eqs. (19) and (20) from the wave function (18) can be repeated when two identical spin $1 / 2$ particle are considered. The configuration space is now the product space spanned by the 20 coordinates given by the 8 space-time coordinates and the 12 angular coordinates of the two particles. To clarify the source of EPR quantum correlations, we consider here two cases: a) the two particles have opposite spin along the $z$-axis; b) the two particles are in the EPR state. In the quantum notation, case (a) corresponds to the spin product state $|\uparrow\rangle|\downarrow\rangle$ and case (b) to the entangled state $(1 / \sqrt{2})(|\uparrow\rangle|\downarrow\rangle-|\downarrow\rangle|\uparrow\rangle)$.

2 Beside the center of mass velocity, also the velocity $v_{e}$ of the center of energy given by $\tanh \chi=v_{e} / c$ is small in the nonrelativistic limit. 


\section{1. (a) The two opposite spins}

The wave function of the state $|\uparrow\rangle|\downarrow\rangle$ is easily written by taking the product of the two terms on the right of Eq. (18) and $S$ and $R_{W}$ are then calculated from modulus and phase of this wave function (for details see Ref. ([23]). The result is

$$
\begin{aligned}
S & =S^{(A)}\left(\boldsymbol{r}_{A}, \theta_{A}\right)+S^{(B)}\left(\boldsymbol{r}_{B}, \theta_{B}\right) \\
R_{W} & =R_{W}^{(A)}\left(\boldsymbol{r}_{A}, \theta_{A}\right)+R_{W}^{(B)}\left(\boldsymbol{r}_{B}, \theta_{B}\right)
\end{aligned}
$$

where $S^{(A, B)}\left(\boldsymbol{r}_{A, B}, \theta_{A, B}\right)$ and $R_{W}^{(A, B)}\left(\boldsymbol{r}_{A, B}, \theta_{A, B}\right)$ are given by Eqs. (19) calculated for particle A and B separately ${ }^{3}$. From Eqs. $(21,22)$ we see that in this case the particles have independent motions. In particular, the Weyl curvature reduces to the sum of the two Weyl curvatures so that each particle is affected only by its own self-force.

\section{2. (b) The two spin EPR state}

The same procedure can be applied to the EPR state of the two spins. The result is [23]

$$
\begin{aligned}
S=\hbar\left[\frac{\gamma_{A}+\gamma_{B}}{2}\right. & +\arctan \left(\csc \frac{\beta_{A}-\beta_{B}}{2} \sin \frac{\beta_{A}+\beta_{B}}{2} \tan \frac{\alpha_{B}-\alpha_{A}}{2}\right)+ \\
& \left.+\arg \left(w_{\uparrow}^{(A)}\left(\boldsymbol{r}_{A}, t\right)\right)+\arg \left(w_{\downarrow}^{(B)}\left(\boldsymbol{r}_{B}, t\right)\right)\right]
\end{aligned}
$$

and

$$
R_{W}=\frac{22}{5 a^{2}\left(1-\cos \beta_{A} \cos \beta_{B}-\cos \Delta \alpha \sin \beta_{A} \sin \beta_{B}\right)}+R_{W}^{(A)}\left(\boldsymbol{r}_{A}, t\right)+R_{W}^{(B)}\left(\boldsymbol{r}_{B}, t\right) .
$$

In this case, although the particle motions are independent in space-time, they are still coupled by the Weyl curvature in the angular variables and, beside the self-force, the particles exert a force on each other. As we have shown elsewhere by considering in detail the behavior of the two particles on a Stern-Gerlach apparatus [23], the features of this geometrical interaction reproduce all results of the standard quantum mechanics and, in particular, violate Bell's inequalities.

\section{Conclusions}

We proposed an alternative approach to quantum mechanics of spin $1 / 2$ particles based on conformal Weyl-gauge invariance. No quantization rules appear explicitly and quantum effects are derived from the existence of a force from Weyl's scalar curvature of the configuration space of the system. A scalar wave function is introduced as a useful ansatz to solve the nonlinear equations of the theory. The particle mass is replaced by Weyl's scalar curvature field and a constant mass appears only at the level of wave equation. In the case of spin $1 / 2$ a one-to-one correspondence is established between the results of the theory and the results of the standard quantum mechanics based on Dirac's spinors and on the second-order Dirac equation [11]. However, the underlying Weyl-gauge symmetry is lost when standard quantum mechanics is exploited. Beside space-time coordinates, internal variables are introduced corresponding to orientational degrees of freedom. These extra coordinates are hidden to experiment and are absent in the standard quantum mechanics, which is based on space-time degrees of freedom only. However, the internal hidden variables and Weyl's geometry of the configuration space are the source of quantum effects, including entanglement. The occurrence of EPR paradox, in particular, can be traced back to a geometrical nonlocal interaction among the particles due to Weyl's curvature. At the present stage we cannot say if this interaction is superluminal, because we studied the EPR state in the nonrelativistic approximation. Future work will be addressed in this direction.

3 Except for a common factor due to the changed dimensionality of the configuration space. 


\section{References}

[1] Belinfante F 1951 A Survay of Hidden Variable Theories (Englewood Cliffs, NJ: Prentice Hall)

[2] Madelung E 1926 Quantentheorie in hydrodynamischer Form Z. Phys. 40332

[3] Bohm D 1952 A suggested interpretation of the quantum theory in terms of "hidden" variables I Phys. Rev. 85166

[4] Bohm D 1952 A suggested interpretation of the quantum theory in terms of "hidden" variables II Phys. Rev. 85180

[5] Fényes I 1952 Eine wahrscheinlichkeitstheoretische Begrundung und Interpretation der Quantenmechanik $Z$. Phys. 13281

[6] Nelson E 1966 Derivation of the Schrödinger equation from Newtonian mechanics Phys. Rev. 1501079

[7] Santamato E 1984 Geometric derivation of the Schrödinger equation from classical mechanics in curved Weyl spaces Phys. Rev. D 29216

[8] Santamato E 1984 Statistical interpretation of the Klein-Gordon equation in terms of the space-time Weyl curvature J. Math. Phys. 252477

[9] Santamato E 1985 Gauge-invariant statistical mechanics and average action principle for the Klein-Gordon particle in geometric quantum mechanics Phys. Rev. D 322615

[10] De Martini F and Santamato E 2011 Derivation of Dirac's equation from conformal differential geometry Quantum Foundations ed A Khrennikov (Melville: AIP) (Preprint quant-ph/1107.3168v1)

[11] Santamato E and De Martini F 2013 Derivation of the Dirac equation by conformal differential geometry Found. Phys. to appear

[12] Bell J S 1987 Speakable and Unspeakable in Quantum Mechanics (Cambridge, UK: Cambridge Univ. Press)

[13] Redhead M 1987 Incompleteness, Nonlocality and Realism: a Prolegomenon to the Philosophy of Quantum Mechanics (Oxford: Clarendon Press)

[14] Einstein A, Podolsky B and Rosen N 1936 Can quantum-mechanical description of physical reality be considered complete? Phys. Rev. 47777

[15] Frenkel J 1926 Die Elektrodynamik des rotierenden Elektrons Z. Phys. 37 243-262

[16] Thomas L H 1926 The motion of the spinning electron Nature 117514

[17] Hanson A J and Regge T 1974 The relativistic spherical top Ann. Phys. (NY) 87 498-566

[18] Souriau J M 1969 Structure des systèmes dynamiques (Paris: Dunod Université)

[19] Corben H C 1968 Classical and quantum theory of spinning particles (San Francisco: Holden-Day)

[20] Sudarshan E C G and Mukunda N 1974 Classical dynamics: a modern perspective (New York: John Wiley \& Sons)

[21] Landau L D and Lifshitz E M 1960 Relativistic Quantum Theory (New York: Pergamon Press)

[22] Brown L M 1958 Two-component fermion theory Phys. Rev. 111(3) 957-964

[23] Santamato E and De Martini F 2012 Solving the quantum nonlocality enigma by Weyl's conformal geometrodynamics Preprint arXiv:1203.0033v1 [quant-ph], submitted to Found. Phys. 